\title{
On the Identification of Changing Tastes
}

\author{
Maximilian Mihm* Kemal Ozbek ${ }^{\dagger}$
}

May 10, 2019

\begin{abstract}
In a dynamic choice environment, an agent's tastes may change over time (e.g., due to present bias, habit formation, or reference dependence). These changes are often systematic with important welfare and policy implications. In this paper, we propose a framework to identify an agent's anticipation about how her preferences change over time and provide a heuristic measure that can inform the design of economic policies. In particular, our method resolves identification issues arising in the previous literature by using the intuitive idea that, while tastes can change, rankings are often monotone with respect to a suitable dominance relation.
\end{abstract}

Key words: changing tastes, consistent planning, dynamic choice, dominance relation.

\section{Introduction}

Many economic activities involve dynamic decision-making, where choices made each period are based on rankings that change over time. ${ }^{1}$ These changes are often systematic and impact welfare by inducing harmful behaviors such as under-saving, addiction, or procrastination (see, e.g., DellaVigna [2009]; Spiegler [2011]; O’Donoghue and Rabin [2015]). To help mitigate such detrimental effects, various policy instruments have been implemented. For instance, there are programs that aim to promote saving behavior (e.g., subsidized saving accounts), regulations that try to reduce consumption of addictive substances (e.g., minimum alcohol pricing), or investment accounts that target to overcome myopic behavior (e.g., opt-in defaults in retirement plans). However, designing such policies, predicting their impact, and evaluating their success, can be challenging especially in a dynamic choice environment with changing tastes. The reason is that proposing an effective policy requires not only identifying how an individual values alternatives today, but also how she anticipates her tastes evolving over time.

\footnotetext{
*New York University Abu Dhabi. Email: max.mihm@nyu.edu.

${ }^{\dagger}$ University of St Andrews. Email: kemal.ozbek@st-andrews.ac.uk.

${ }^{1}$ For instance, in consumption-saving problems, various behavioral biases can lead to changes in tastes over time, including present-bias (e.g., Laibson [1997]; O'Donoghue and Rabin [1999]), addiction (e.g, Becker and Murphy [1988]), or status-quo bias (e.g., Thaler and Benartzi [2004]).
} 
To illustrate an instance of such a policy challenge, consider the Saving Gateway program, which is an individual development account that was proposed in the UK. ${ }^{2}$ The objective of this program was to promote engagement with financial services among low income households, thereby encouraging the development of a saving habit for rainy days (Kempson et al. [2005]). In order to incentivize participation, the government proposed to offer saving accounts where personal contributions would be matched with public funds. For this, the government needed to identify suitable policy parameters (e.g., match-rate or duration), which was especially challenging given that preferences over saving programs could change over time. As such, the government consulted experts on the design of saving policies. Based on a wide range of expert opinions, several pilot projects were commissioned, which provided also the opportunity to assess the desirability of programs. ${ }^{3}$ However, despite evidence of potential benefits, a full-scale version of the pilot projects was found to be too costly and, ultimately, the saving program was not implemented (Emmerson et al. [2007]). ${ }^{4}$ On the other hand, ex-post surveys showed that the willingness to participate in a saving program would have been high, even with lower parameters. How could policy-makers have identified the willingness to participate ex-ante, in order to design pilot projects that would provide better information about cost effective yet incentivizing programs?

In this paper, we propose a framework to identify an agent's anticipation about how her preferences change over time and provide a simple measurement procedure that can be used to inform the design of policy instruments. Our identification assumption is that the set of outcomes in each period is endowed with a dominance relation, whereby some alternatives can be "objectively" ranked. For instance, while an individual's relative taste for different aspects of a saving policy (such as its duration or match-rate) may change over time, it is natural to assume that the individual will prefer a policy that is superior in every dimension. In Section 2, we illustrate, in a two-period setting, how this identification assumption allows us to elicit the anticipated future rankings over saving policies. In particular, we demonstrate with the example how the dominance relation helps to overcome identification problems that arise in the prior literature on changing tastes, in particular Gul and Pesendorfer [2005] (henceforth, GP).

Our formal analysis extends the insights developed in the two-period example to a general finitehorizon framework where, in each period $t$, the agent makes a decision that determines an outcome for period $t$ and a continuation problem for period $t+1$. Following GP, our primitive is the agent's period 0 preferences over dynamic decision problems in an environment without uncertainty. ${ }^{5}$ In

\footnotetext{
${ }^{2}$ The UK government proposed the Saving Gateway program in the early 2000s. Key policy parameters of the program included the duration (i.e., number of years individuals could participate) and the match-rate (i.e., ratio at which personal savings would be matched with public), as well as caps on monthly contributions and restrictions on withdrawals (see, e.g., Kempson et al. [2005]).

${ }^{3}$ For instance, experts suggested a wide-range of match-rates from $10 \%$ to $100 \%$, durations from 30 to 60 months, and monthly contribution caps from $£ 10$ to $£ 125$.

${ }^{4}$ The program was initially targeted to reach 8 million low-income households who traditionally had accumulated very few savings. With imprecise estimates from experts, policy-makers ran pilot projects with a variety of parameters, and essentially settled on the mid-point parameters for the final program. Despite the findings in the pilot projects, which cost more than $£ 20$ million to conduct, the Saving Gateway program was scrapped in 2010. However, after a further pilot project, a similar program, Help to Save, was re-proposed in 2018.

${ }^{5} \mathrm{~A}$ growing literature also studies anticipated learning dynamics in environments with uncertainty concerning the
} 
particular, we focus on a consistent planner who equates each choice problem in period 0 with a path of future choices consistent with her anticipated rankings over continuation problems (e.g., Strotz [1956]; Pollak [1968]). As such, the agent's preferences in period 0 satisfy Axiom 2 (no-compromise), which is a rationality condition introduced in GP. ${ }^{6}$ We show that, together with Axiom 1 (weak order), no-compromise characterizes the period 0 behavior of a consistent planner, generalizing GP's axiomatic characterization from their finite-outcome setting to an environment with arbitrarily large set of outcomes (Proposition 1). This result provides a useful benchmark for our subsequent analysis since it indicates that identification problems, which arise in GP's finite-outcome setting, cannot be resolved by simply expanding the set of outcomes.

To identify the anticipated future rankings of a consistent planner, we equip the set of outcomes in each period with a dominance relation. Such dominance relations are common in applied models of changing tastes (e.g., vector-dominance in consumer choice problems). Our first main result (Theorem 1) introduces an intuitive condition (Axiom 3) that characterizes the class of regular consistent preferences for which anticipated rankings are upper semicontinuous and monotone in the dominance relation. Our second main result (Theorem 2) shows that, for regular consistent preferences, anticipated rankings are unique. In fact, we provide an explicit procedure (Definition 1) for how today's preferences can be used to elicit these future rankings. Our third main result (Theorem 3), shows that the identification of anticipated rankings is robust to the choice of dominance relation. Finally, we discuss how the dominance relation can be used to obtain heuristic welfare measurements and characterize potential tie-breaking rules.

The remainder of the paper is organized as follows. Section 2 provides our motivating example. Section 3 introduces the dynamic choice framework and a non-parametric model of consistent planning. Section 4 provides our main results, and an application to a consumption-saving problem. Section 5 discusses related literature. Proofs are given in an Appendix.

\section{Motivating example}

To motivate our analysis, we consider an example based on the Saving Gateway program discussed in the Introduction. We first illustrate some identification problems that arise in the previous literature, and then discuss how our approach remedies these problems, thereby avoiding misguided policy interventions. The example also suggests how our general framework can be used to heuristically measure anticipated well-being, which could be a relevant input for policy analysis.

Policy problem. A policy-maker (PM) wants to encourage an individual to save more in order to help the individual develop a saving habit over time. There is a status-quo saving policy $q=\left(d_{q}, m_{q}\right)$, but the individual does not find it attractive enough to participate. The PM therefore wants to state of the world or the agent's tastes (see Section 5).

${ }^{6}$ Informally, Axiom 2 states that for any two decision problems, the agent will always be indifferent between their union and one of the two problems. In particular, no-compromise implies that the agent has a desire for commitment in period 0, which could exclude some learning dynamics that induce a strict desire for flexibility (see Section 5). 
provide a new saving policy $s=\left(d_{s}, m_{s}\right)$, which would differ in duration-time $d$ and match-rate $m$, to incentivize the individual to participate. The ideal policy should not only be effective (maximizing the incentives for the individual), but also should be affordable (minimizing the costs to the taxpayers). The PM acknowledges that although the costs of a chosen policy might be born today, the policy will not be implemented until tomorrow. As such, the effectiveness of a policy would depend on how the individual anticipates valuing the policy in the future, and not necessarily on how she values the policy today. As a result, the PM needs to understand today the individual's anticipated future rankings in addition to the costs of providing the policy. With these objectives in mind, what kind of saving policy should the PM provide?

To choose a policy, suppose that the PM consults an expert. The expert suggests that some policy $x=\left(d_{x}, m_{x}\right)$, where $d_{x}<d_{q}$ and $m_{x}>m_{q}$, should be a candidate policy to consider. In particular, the expert claims that the individual will prefer $x$ over $q$ in the future (i.e., $x \succsim^{*} q$ ) since (i) surveys suggest that today $x$ is a comparable policy to $q$ (i.e., $\{x\} \succsim\{q\}$ ), and (ii) as time goes by, the individual will value the match-rate of a policy more than the duration. To decide whether to implement policy $x$, the PM would like to verify the expert's claim, but how?

Choice experiments. To answer this question, the previous literature (in particular, GP) suggests considering preferences over choice sets (i.e., choice experiments), which provide either commitment today or choice between policies in the future. Specifically, looking at the individual's preferences today $\succsim$ over $\{x\},\{q\}$, and $\{x, q\}$ may allow the PM to make inferences about tomorrow's anticipated ranking of policies $x$ and $q$ (see Eq. (1) in Section 3.3).

To illustrate, suppose that the PM observes $\{x\} \sim\{x, q\} \succ\{q\}$. In that case, GP interprets that the individual anticipates choosing $x$ from $\{x, q\}$ tomorrow, and infer from this observation that the individual anticipates ranking policy $x$ above $q$; that is, $x \succ^{*} q$. As such, the PM might want to find an alternative policy $x^{\prime}$, which is less costly than $x$ and yet provides enough incentives for the individual to participate. One way to find such an alternative policy $x^{\prime}$ could be to use the particular structure of the policy space, and lower the duration and match-rate of policy $x$ while ensuring that this alternative policy remains better than $q$ (i.e., $x^{\prime} \succsim^{*} q$ ).

However, lowering duration-time and match-rate could be a misguided policy intervention because GP's definition can lead to misidentification of the anticipated ranking between $x$ and $q$. For instance, the observation $\{x\} \sim\{x, q\} \succ\{q\}$ is also consistent with the individual anticipating a choice of $x$ from $\{x, q\}$ while being indifferent; that is, $x \sim^{*} q$. In that case, since it would be $x \succ^{*} x^{\prime}$ (and therefore $q \succ^{*} x^{\prime}$ ), the new policy $x^{\prime}$ would be ineffective. In fact, when $x \sim^{*} q$, the correct policy could be to raise duration-time and match-rate of policy $x$ to obtain some $x^{\prime \prime}$, in order to guarantee that the newly offered policy provides enough incentives for the individual to participate.

Alternatively, the PM may observe $\{x\} \sim\{x, q\} \sim\{q\} .{ }^{7}$ In this case, GP infer that the individual anticipates being indifferent between policy $x$ and $q$; that is, $x \sim^{*} q$. As a result, the PM may want to increase the duration and match-rate to obtain an alternative policy $x^{\prime \prime}$, which incentivizes the

\footnotetext{
${ }^{7} \mathrm{~A}$ third possibility is to observe $\{x\} \succ\{x, q\} \sim\{q\}$. In this case, GP infer that $q \succ{ }^{*} x$, and so the policy the expert proposes would be found ineffective (see Eq. (1) in Section 3.3).
} 
individual to participate. However, this may be also a misguided policy decision. For instance, the observation that $\{x\} \sim\{x, q\} \sim\{q\}$ can also be consistent with the individual anticipating to strictly rank policy $x$ over $q$ in the future, even though she is indifferent between these policies today; that is, $x \succ^{*} q$. In that case, instead of considering policy $x^{\prime \prime}$, which has a higher cost, the PM should consider a cheaper policy $x^{\prime}$ that has a lower duration and match-rate than $x$, while still being better than $q$ (i.e., $x^{\prime} \succ^{*} q$ ).

Discussion. Is there a way to address these limitations, and provide a better guidance on policy interventions? For instance, suppose the PM observes that $\{x\} \sim\{x, q\} \succ\{q\}$. As discussed above, this observation indicates that the individual anticipates choosing $x$ from $\{x, q\}$ tomorrow, and so the PM can infer that $x \succsim^{*} q$. To make a well-informed policy choice, however, the PM needs to understand if this anticipated ranking between $x$ and $q$ is weak or strict. In general, since indifferences are a knife-edge case, the PM may think it is unlikely that the individual would be indifferent between two arbitrary policies. However, there may be greater reason for the PM to be concerned about indifferences when policies are chosen deliberately (e.g., when $x$ is chosen by an expert). ${ }^{8}$ Yet, there is not enough information for the PM to distinguish between a weak and strict ranking by considering only choices between $\{x\},\{q\}$, and $\{x, q\}$, and the PM would therefore need additional data on choice experiments. For example, if $x \succ^{*} q$, then intuitively it should be possible to find another policy $z$ that "separates" $x$ from $q$, such that $x \succsim^{*} z \succ^{*} q$. However, to ensure that $z$ actually helps to solve the identification problem, the PM would need to be confident that the ranking between $z$ and $q$ is strict.

Our approach to find such a policy is based on a simple intuition. First, if one policy dominates another policy (i.e., has higher duration and match-rate), then the individual should anticipate ranking it higher. Second, if an adjustment made to a policy is sufficiently small, then the individual's anticipated ranking should not change much. As such, the fact that the policy space is rich and equipped with a natural dominance relation can provide a simple solution to the misidentification problem. Moreover, with a rich data set, our identification approach can be used to elicit the agent's anticipated rankings and thereby pin-down her behavioral parameters (see, e.g., Section 4.4).

In addition to the resolution of misidentification problems discussed above, our identification approach suggests a heuristic measure for the strength of an individual's anticipated rankings. In particular, since the dominance relation provides an objective way to rank some alternatives, the PM can understand how strong or weak anticipated rankings are, and thereby measure anticipated wellbeing. For instance, if $x \succ^{*} q$, then the PM could look for the highest $\epsilon$ such that $0<d_{z}-d_{q}<\epsilon$ and $0<m_{z}-m_{q}<\epsilon$, while also $x \succ^{*} z$. A high $\epsilon$ could suggest that the anticipated ranking of $x$ above $q$ is strong, while a low $\epsilon$ could suggest that the anticipated ranking is weak. Although such a heuristic measurement will not be complete, a PM who needs to balance the benefits and costs of a saving program may still find it as a useful input into the policy design problem (see Section 4.3).

\footnotetext{
${ }^{8}$ In particular, when the expert chooses a policy proposal $x \succsim^{*} q$, while also trying to minimize its costs, then the expert is naturally led to make a proposal that satisfies indifference. In Section 3.3, we discuss further reasons to be concerned about anticipated indifferences in dynamic choice environments.
} 
Extending the ideas developed in this example, our formal analysis contributes to the literature on changing tastes by providing a solution to two important identification challenges. First, we develop a framework that has sufficient structure to uniquely identify anticipated rankings, and yet is general enough to encompass most applied models of changing tastes. Second, we propose a specific procedure to elicit anticipated future rankings, and measure anticipated well-being, based on observable choice data.

\section{Consistent planning}

In this section, we present a general finite-horizon dynamic choice framework and define a nonparametric model of consistent planning. We then provide a characterization result, which extends GP's axiomatization of the consistent planning model, and we discuss limitations of GP's identification approach. These discussions provide a useful benchmark for our main results in Section 4 .

\subsection{Framework}

We consider a finite-horizon decision framework. In each period $t=0, \ldots, T$ there is a set of possible outcomes $X_{t}$, with typical elements denoted $x, y, z$. For any non-empty set $Z$, we denote by $K(Z)$ the collection of all non-empty finite subsets of $Z$. Following GP, we define decision problems recursively. For the last period, let $\mathcal{X}_{T}=K\left(X_{T}\right)$ be the set of period $T$ decision problems. For periods $1 \leq t<T$, define recursively the set of period $t$ decision problems as $\mathcal{X}_{t}=K\left(X_{t} \times \mathcal{X}_{t+1}\right)$, with typical elements denoted by $a, b, c$. Each $a \in \mathcal{X}_{t}$ is a finite menu of alternatives of the form $(x, b)$, where $x \in X_{t}$ is the outcome in period $t$ and $b \in \mathcal{X}_{t+1}$ is the continuation problem in period $t+1$. We set $X_{0}=\left\{x_{0}\right\}$ and call $X_{0} \times \mathcal{X}_{1}$ the set of period 0 alternatives. A decision problem with a singleton continuation problem in each period can be identified with a unique outcome path $x^{T}=\left(x_{0}, . ., x_{T}\right)$. Let $X=X_{0} \times \ldots \times X_{T}$ be the set of possible outcome paths.

For any $0 \leq t<T$, a $t$-period history is a $(t+1)$-tuple of outcomes $\left(x_{0}, \ldots, x_{t}\right)$, where $x_{k} \in X_{k}$ for $0 \leq k \leq t$. Let $H_{t}$ denote the set of all $t$-period histories and let $H=\cup_{t=0}^{T-1} H_{t}$ be the set of all histories. For a history $h=\left(x_{0}, \ldots, x_{t}\right) \in H_{t}$ we denote the length of history $h$ by $|h|=t+1$. We often denote an alternative $\left(x_{0}, a\right) \in X_{0} \times \mathcal{X}_{1}$ by $(h, b) \in H_{t} \times \mathcal{X}_{t+1}$ where $h \in H_{t}$ is the longest history which is common to all outcome paths in $\left(x_{0}, a\right){ }^{9}$

For $h \in H_{t-1}$ and $a \in \mathcal{X}_{t}$, we call a pair $(h, a)$ a (decision) node at period $t$. Let $N_{t}$ denote the set of all nodes at period $t$ and $N=\cup_{t=1}^{T} N_{t}$ be the set of all nodes with generic elements denoted by $n, n^{\prime}, n^{\prime \prime}$. Note that we can identify $N_{0}$ with $X_{0} \times \mathcal{X}_{1}$, and we therefore often refer to a period 0 alternatives $\left(x_{0}, a\right)$ as an initial node.

A plan is a map $\varphi: N \rightarrow N \cup X$ that assigns each node $n=(h, a) \in N_{t}$ either to a node $n^{\prime}=\left(h, x_{t}, b\right) \in N_{t+1}$ when $t<T$ such that $\left(x_{t}, b\right) \in a$, or to an outcome path $\left(h, x_{T}\right) \in X$ when $t=T$ such that $x_{T} \in a$. Let $\Phi$ denote the set of all plans. For any plan $\varphi \in \Phi$ and $k=0, \ldots, T$ define

\footnotetext{
${ }^{9}$ Formally, $h \in H_{t}$ is the longest history such that for each $0 \leq k \leq t,\left(h_{k}, b_{k+1}\right) \in b_{k}$ for some $b_{k} \in \mathcal{X}_{k}$ with $b_{k+1}=b$ and $b_{1}=a$. Note that for each decision problem $a \in \mathcal{X}_{1}$, such a representation must be unique.
} 
$\varphi^{k}: N \cup X \rightarrow N \cup X$ as $\varphi^{k}(x)=x$ for any $x \in X$ and for any $n \in N, \varphi^{0}(n)=n, \varphi^{1}(n)=\varphi(n)$ and $\varphi^{k+1}(n)=\varphi^{k}(\varphi(n))$. In particular, $\varphi^{T}(n)$ denotes the outcome path induced by $\varphi$ given node $n$.

For a given history $h \in H$, we call a complete and transitive binary relation $\succsim_{h}$ defined over the set $X_{|h|} \times \mathcal{X}_{|h|+1}$ a history-dependent ranking, and denote its asymmetric part by $\succ_{h}$ and symmetric part by $\sim_{h}$. We call a given collection of history-dependent rankings, $\left(\succsim_{H}\right) \equiv\left(\succsim_{h}\right)_{h \in H}$, a ranking system.

For any decision node $n=(h, a) \in N$, we denote the set of feasible decision nodes that are optimal for the ranking $\succsim_{h}$ by $M_{\succsim_{h}}(a) \equiv\left\{(h, x, b) \in N_{|h|}:(x, b) \in a\right.$ and $\left.(x, b) \succsim_{h}(y, c), \forall(y, c) \in a\right\} .{ }^{10}$ We say that a plan $\varphi$ is rationalized by the ranking system $\left(\succsim_{H}\right)$ if, for each ranking $\succsim_{h}$ in $\left(\succsim_{H}\right)$, $\varphi(n) \in M_{\succsim h}(a)$ at each decision node $n=(h, a) \in N$. We denote the set of all plans that can be rationalized by the ranking system $\left(\succsim_{H}\right)$ by $\Phi\left(\succsim_{H}\right)=\left\{\varphi \in \Phi: \varphi(n) \in M_{\succsim_{h}}(a), \forall n=(h, a) \in N\right\} .{ }^{11}$

\subsection{Consistent preferences}

Our primitive is a binary relation $\succsim$ on $X_{0} \times \mathcal{X}_{1}$, which we interpret as the agent's preference relation over initial nodes. We denote strict preference by $\succ$, indifference by $\sim$, and denote by $\nsim$ the negation of the indifference relation. We focus in the remainder of the paper on a preference relation $\succsim$ for which the commitment ranking over outcomes paths is a weak order (i.e., the restriction of $\succsim$ to $X$ is complete and transitive).

An agent is a planner if her preferences $\succsim$ are compatible with a plan $\varphi \in \Phi$; that is, for each node $n=\left(x_{0}, a\right)$, the agent's preference relation $\succsim$ satisfies $\left(x_{0}, a\right) \sim \varphi^{T}(n)$. As such, a planner equates each decision problem in period 0 with an outcome path induced by her plan $\varphi$. We say that the agent is a consistent planner if her plan $\varphi$ can be rationalized by a ranking system $\left(\succsim_{H}\right)$; that is $\varphi \in \Phi\left(\succsim_{H}\right)$ for some ranking system $\left(\succsim_{H}\right)$. In that case, we also say that $\succsim$ can be rationalized by the ranking system $\left(\succsim_{H}\right)$, and call $\succsim$ a consistent preference.

The following axioms are satisfied by a consistent preference $\succsim$.

Axiom 1. [Weak order] For all $h \in H$, and $a, b, c \in \mathcal{X}_{|h|}$, (i) $(h, a) \succsim(h, b)$ or $(h, b) \succsim(h, a)$, and (ii) $(h, a) \succsim(h, b)$ and $(h, b) \succsim(h, c)$ implies $(h, a) \succsim(h, c)$.

Axiom 2. [No-compromise] For all $h \in H$, and $a, b \in \mathcal{X}_{|h|},(h, a \cup b) \sim(h, a)$ or $(h, a \cup b) \sim(h, b)$.

Axiom 1 is a standard consistency postulate, requiring that preferences are complete and transitive. Axiom 2 reflects the idea that the agent anticipates a specific alternative to be chosen in each period, and cares only about the resulting outcome path.

The following proposition provides a useful benchmark for our later analysis by extending GP's characterization of consistent preferences from their finite-outcome setting to a more general environment.

Proposition 1. $\succsim$ is a consistent preference if and only if it satisfies Axioms 1 and 2.

\footnotetext{
${ }^{10}$ The set $M_{\succsim_{h}}(a)$ is non-empty because $\succsim_{h}$ is a weak order and $a$ is finite.

${ }^{11}$ In general, $\Phi\left(\succsim_{H}\right)$ may contain more than one plan.
} 
Proposition 1 characterizes the preferences of an agent who acts as if she anticipates solving a consistent planning problem. In particular, the no-compromise axiom implies that the agent anticipates following a certain plan that can be rationalized by a ranking system. However, the ranking system rationalizing the plan may not be unique. ${ }^{12}$

In particular, there are two reasons why the ranking system may not be uniquely identified. First, the proof of Proposition 1 uses the Hausdorff Maximal Principle to extend anticipated rankings from finite choice environments to arbitrary environments. Second, the rankings in finite environments are obtained by using GP's canonical ranking, which, however, as we discuss below, can misidentify the anticipated rankings. ${ }^{13}$

\subsection{Indifferences and canonical rankings}

The primary source of misidentification in GP's approach is the presence of indifferences in anticipated future rankings. Below, we first argue that indifferences can naturally arise in many dynamic choice problems, and then describe how GP's canonical ranking can lead to misidentification.

Indifferences. In dynamic choice environments, indifference conditions frequently occur in an endogenous way, simply because the agent - as a planner - anticipates making equivalent decisions in future choice problems. For instance, in the context of consumption-saving problems (e.g., Strotz [1956]; Phelps and Pollak [1968]; Laibson [1997]), rational addiction models (e.g., Becker and Murphy [1988]; Becker et al. [1994]), or investment problems (e.g., Thaler and Benartzi [2004]; Barberis and Xiong [2009]), there are generally non-binding constraints on continuation problems such that the agent remains indifferent when they are removed.

On the other hand, indifferences also arise for exogenous reasons, especially in multi-player settings (e.g., principal-agent problems). For instance, to find a cost-effective saving scheme, a policy-maker may look for various options that will make the individual indifferent to a status quo in the future (see Section 2); to measure intertemporal substitution rates, an analyst may want to design an experiment eliciting an agent's anticipated willingness-to-pay (see Section 4.4) ${ }^{14}$; or, to achieve a fair division in a dynamic contracting problem, a principal may want to design a contract such that the agent's individual rationality and/or incentive compatibility constraints in the future are satisfied with equality (see, e.g., Spiegler [2011]). As a result, indifferences often play a key role in applied and experimental settings, and it can be important to identify whether an agent anticipates being indifferent or not. ${ }^{15}$

\footnotetext{
${ }^{12}$ For instance, the overwhelming temptation preferences in Gul and Pesendorfer [2001] satisfy Axioms 1 and 2, and are therefore consistent preferences. Yet there can be more than one ranking system that rationalizes the same overwhelming temptation preference (see Section 5).

${ }^{13}$ Our main characterization result in Section 4.2 does not directly use Proposition 1, and instead provides an entirely different proof method that is based on a constructive argument.

${ }^{14}$ Likewise, in order to measure risk aversion in the future, the analyst may need to elicit certainty equivalents, which are also defined in terms of indifference conditions.

${ }^{15}$ For instance, distinguishing between recursive models of consistent planning (Phelps and Pollak [1968]) or selfcontrol (Gul and Pesendorfer [2004]) requires identifying whether anticipated rankings are strict or weak (see, e.g., Toussaert [2018]).
} 
Canonical rankings. To describe the identification approach in GP, we can focus on a two-period framework similar to the example in Section 2 . Let $Z$ be a set of outcomes in period 1 , and let $\succsim$ be a consistent preference on $K(Z)$, interpreted as the agent's preferences in period 0 over menus of alternatives in period 1 . GP define a canonical ranking $\succsim_{1}^{G P}$, which they interpret as the agent's anticipated ranking in period 1 . In particular, for all $x, y \in Z,{ }^{16}$

$$
\begin{aligned}
& x \succ_{1}^{G P} y \text { if }\left\{\begin{array}{c}
\{x\} \sim\{x, y\} \nsim\{y\} \\
\text { or } \\
\exists z \in Z,\{x, z\} \sim\{x\} \sim\{y\} \nsim\{y, z\}
\end{array} \quad\right. \text {, and } \\
& x \sim_{1}^{G P} y \text { if } \quad \forall z \in Z,\{x, z\} \sim\{x\} \Leftrightarrow\{y\} \sim\{y, z\} .
\end{aligned}
$$

From the definition, it follows that, whenever the agent is not indifferent between committing to one of the two alternatives $x$ or $y$ today (i.e., $\{x\} \nsim\{y\}$ ), then she always anticipates having a strict preference between these alternatives tomorrow (i.e, $x \nsim_{1}^{G P} y$ ). However, as we illustrate in a consumption-saving example with present bias, many applications of consistent planning violate this restriction (see Section 4.4).

On the other hand, as we show in Section 2, GP's canonical ranking can (i) predict a strict preference between $x$ and $y$ tomorrow, even though the agent actually anticipates being indifferent, or (ii) predict indifference between $x$ and $y$ tomorrow, even though the agent actually anticipates having a strict preference. To address such identification problems, we extend on the intuition developed in Section 2 by considering a richer outcome-space equipped with a dominance relation. In particular, in the following section, we show how the anticipation of a strict ranking between $x$ and $y$ can be revealed by finding a dominating alternative $z$ that is close to $y$.

\section{Regular consistent preferences}

In this section, we show when consistent preferences can be rationalized uniquely by a ranking system, and how these rankings can be elicited robustly from today's preferences. For this, we equip the set of outcomes in each period with a dominance relation, which satisfies a number of natural properties. We then discuss how this richer framework can be used to obtain heuristic measures, represent future rankings, and characterize tie-breaking rules. Finally, we illustrate how to apply our elicitation procedure in a consumption problem with present-bias.

\subsection{Regularity}

We now assume that, in each period $t$, the outcome set $X_{t}$ is a topological space equipped with a dominance relation $\triangleright_{t}$ satisfying three properties: (i) [strict partial order] $\triangleright_{t}$ is irreflexive and transitive; (ii) [local non-satiation] for any $x \in X_{t}$ and any open set $O_{x} \subset X_{t}$ containing $x$,

\footnotetext{
${ }^{16}$ The definition of the canonical rankings in Eq. (1) is not identical but equivalent to the definition given in Gul and Pesendorfer [2005, pp. 444-445].
} 
there exists some $y \in O_{x}$ such that $y \triangleright_{t} x$; and (iii) [upper semicontinuity] for any $x \in X_{t}$, the set $\left\{y \in X_{t}: x \triangleright_{t} y\right\}$ is open. For instance, when outcomes are consumption-bundles, statecontingencies, or lotteries, the Euclidean metric can be used to define the topology. Respectively, strict vector comparison, state-wise outcome dominance, and first-order-stochastic dominance can be used to define the dominance relation. In most applications, these dominance relations are strict partial orders satisfying local non-satiation and upper semicontinuity. For simplicity, we often drop time-scripts and write $\triangleright$ for the dominance relation in each period.

We say that a consistent preference $\succsim$ is regular if it is $\triangleright$-sensitive and it is compatible with a plan that can be rationalized by a $\triangleright$-regular ranking system. ${ }^{17} \mathrm{~A}$ preference relation $\succsim$ is $\triangleright$-sensitive if, for $h \in H, x, y \in X_{|h|}$ and $a \in \mathcal{X}_{|h|+1}, x \triangleright y$ implies $(h, x, a) \nsim(h, y, a)$; that is, for a given history and continuation problem, when $x$ dominates $y$, the agent is not indifferent between the alternative with $x$ and the alternative with $y$. A ranking system $\left(\succsim_{H}\right)$ is $\triangleright$-regular if, for each $h \in H$, the history-dependent ranking $\succsim_{h}$ satisfies (i) [monotonicity] for all $x, y \in X_{|h|}, x \triangleright y$ implies $(x, a) \succ_{h}(y, a)$ for all $a \in \mathcal{X}_{|h|+1}$, and (ii) [upper semicontinuity] for all $(x, a) \in X_{|h|} \times \mathcal{X}_{|h|+1}$, and for all $b \in \mathcal{X}_{|h|+1}$, the lower contour set $\left\{y \in X_{|h|}:(x, a) \succ_{h}(y, b)\right\}$ is open. These properties are satisfied in many applications of the consistent planning model. In particular, monotonicity implies that the dominance relation provides an unambiguous ranking of some of the alternatives, while upper semicontinuity ensures that a compact set has a maximum.

\subsection{Main results}

In this section, we present our main results: characterization of regular consistent preferences, identification of anticipated rankings, and robustness of the elicitation procedure.

Characterization. Our first objective is to characterize regular consistent preferences. Two additional conditions, which are summarized in the following axiom, are satisfied when a consistent preference is regular.

Axiom 3. [Regularity] For all $h \in H, x, y \in X_{|h|}$, and $a, b \in \mathcal{X}_{|h|+1}$ :

(i) If $x \triangleright y$, then $(h,\{(x, a),(y, a)\}) \nsim(h, y, a)$.

(ii) If $(h,\{(x, a),(y, b)\}) \nsim(h, y, b)$, then for any $x^{\prime} \triangleright x$, there is some $y^{\prime} \triangleright y$ such that $\left(h,\left\{\left(x^{\prime}, a\right),\left(y^{\prime}, b\right)\right\}\right) \nsim\left(h, y^{\prime}, b\right)$.

Part (i) reveals that the agent anticipates her rankings being monotone with respect to the dominance relation $\triangleright$. In particular, if $x$ dominates $y$, then the agent anticipates choosing $(x, a)$ from $\{(x, a),(y, b)\}$ following history $h$, because she anticipates preferring a dominating alternative. Part (ii) reveals that the agent anticipates her rankings being upper semicontinuous. In particular, $(h,\{(x, a),(y, b)\}) \nsim(h, y, b)$ indicates that the agent does not anticipate choosing $(y, b)$ from

\footnotetext{
${ }^{17}$ Recall that $\succsim$ is a consistent preference if there is a plan $\varphi$, rationalized by a ranking system ( $\left.\succsim_{H}\right)$, such that for every node $n=\left(x_{0}, a\right),\left(x_{0}, a\right) \sim \varphi^{T}(n)$. The plan $\varphi$ is rationalized by the ranking system $\left(\succsim_{H}\right)$ if, for any decision node $n=(h, a) \in N, \varphi(n) \in M_{\succsim h}(a)$.
} 
$\{(x, a),(y, b)\}$ after the history $h$. As such, when $x$ is replaced with another alternative $x^{\prime}$ that dominates $x$, then for any alternative that is sufficiently close to $y$ it should be the case that the agent anticipates choosing $\left(x^{\prime}, a\right)$ and not $\left(y^{\prime}, b\right)$. Moreover, at least one such alternative, $y^{\prime}$, should dominate $y$.

In the following, let $\succsim$ be a $\triangleright$-sensitive preference relation.

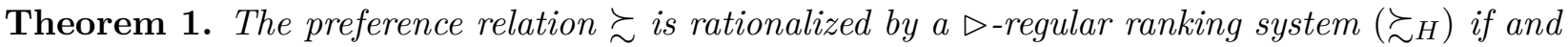
only if $\succsim$ satisfies Axioms 1, 2, and 3 .

Since the ranking system $\left(\succsim_{H}\right)$ that rationalizes $\succsim$ is $\triangleright$-regular, the preference relation $\succsim$ in Theorem 1 is not only consistent but also regular. As a result, Theorem 1 characterizes a subclass of the preferences in Proposition 1. However, our proof of Theorem 1 does not build on Proposition 1. In particular, while the proof of Proposition 1 is non-constructive, the proof of Theorem 1 constructively uses Axiom 3 to show the existence of a ranking system $\left(\succsim_{H}\right)$ rationalizing $\succsim$.

Identification. Theorem 1 characterizes regular consistent preferences; however, it does not show whether a ranking system that rationalizes preferences is unique or can be recovered from choice data. Our second main result shows that a regular consistent preference can, in fact, be rationalized by a unique regular ranking system, which is defined below.

Definition 1. [Revealed ranking system] For a consistent preference $\succsim$, we denote its revealed ranking system by $\left(\succsim_{H}^{*}\right)$ where, for each history $h \in H,(x, a) \succ_{h}^{*}(y, b)$ if there exists $z \triangleright y$ such that $(h,\{(x, a),(z, b)\}) \nsim(h, z, b)$, and $(y, b) \succsim_{h}^{*}(x, a)$ otherwise.

To motivate this definition, suppose the agent anticipates that, following history $h$, she will strictly prefer $(x, a)$ over $(y, b)$. Then, not only should the agent anticipate choosing $(x, a)$ from $\{(x, a),(y, b)\}$, but there should be a nearby alternative $z$ dominating $y$ such that $(x, a)$ is still chosen from $\{(x, a),(z, b)\}$. On the other hand, if such a $z$ cannot be found, then the agent anticipates weakly preferring $(y, b)$ over $(x, a)$ following history $h .^{18}$

In the following, let $\succsim$ be a $\triangleright$-sensitive preference relation and $\left(\succsim_{H}\right)$ be a $\triangleright$-regular ranking system.

Theorem 2. If ranking system $\left(\succsim_{H}\right)$ rationalizes $\succsim$, then $\left(\succsim_{H}\right)=\left(\succsim_{H}^{*}\right)$.

Theorem 2 provides a simple procedure to elicit (uniquely) the future rankings anticipated by a regular consistent planner. As such, the theorem shows how the dominance relation in our framework can be used to operationalize the consistent planning model, in contrast to Proposition 1, which applies in a more general framework but does not achieve identification.

\footnotetext{
${ }^{18}$ Note, in particular, that a strict anticipated ranking can always be revealed with a finite amount of data. However, as usual, identifying indifference would require infinite data.
} 
Robustness. Theorem 2 shows how, using a dominance relation, anticipated future rankings can be elicited uniquely. In general, however, there may be more than one dominance relation in the environment, and it may therefore seem possible to elicit multiple revealed ranking systems. Our third main result shows that the identification procedure in Definition 1 is, in fact, robust to the choice of dominance relation.

In the following, let $\succsim$ be a $\triangleright^{j}$-sensitive preference relation and $\left(\succsim_{H}^{j}\right)$ be a $\triangleright^{j}$-regular ranking system for $j=1,2$, where $\triangleright^{1}$ and $\triangleright^{2}$ refer to two different dominance relations. ${ }^{19}$

Theorem 3. If ranking systems $\left(\succsim_{H}^{1}\right)$ and $\left(\succsim_{H}^{2}\right)$ rationalize $\succsim$, then $\left(\succsim_{H}^{1}\right)=\left(\succsim_{H}^{2}\right)$.

Theorem 3 shows that the elicitation procedure in Definition 1 would lead to the same revealed ranking system regardless of the dominance relation.

\subsection{Discussion}

We now discuss how our framework can be further used to obtain heuristic measures, represent future rankings, and characterize tie-breaking rules, which can be useful in applied settings (see Section 2). In the following, let $\succsim$ be a regular consistent preference.

Heuristic measurements. To define a heuristic measure of the strength of anticipated future rankings, suppose that each period outcome space $X_{t}$ is equipped with a metric $d_{t}$ (as, for example, when $\left.X_{t} \subset \mathbb{R}^{n}\right)$. For two nodes $n=(h, x, a)$ and $n^{\prime}=(h, y, b)$, we want to consider the set of outcomes $z$ that dominate $y$, such that $(x, a)$ is still anticipated to be preferred to $(z, b)$. For this, let $D\left(n, n^{\prime}\right)=\left\{z \in X_{t}: z \triangleright y,(x, a) \succsim_{h}^{*}(z, b)\right\}$, and define $\lambda\left(n, n^{\prime}\right)=\sup _{z \in D\left(n, n^{\prime}\right)} d_{|h|}(z, y)$ if $D\left(n, n^{\prime}\right) \neq \emptyset$, and $\lambda\left(n, n^{\prime}\right)=0$ otherwise.

By Theorem 2, $\lambda$ is a well-defined positive valued function on $N \times N$ and, by Theorem 3, it does not depend on the choice of dominance relation. Note that a high $\lambda\left(n, n^{\prime}\right)$ suggests that the anticipated ranking of $(x, a)$ above $(y, b)$ following history $h$ is strong, while a low $\lambda\left(n, n^{\prime}\right)$ suggests that the anticipated ranking between them is weak. Moreover, the heuristic measure $\lambda$ satisfies (i) $(x, a) \succ_{h}^{*}(y, b)$ if and only if $\lambda\left(n, n^{\prime}\right)>0$, while (ii) $(x, a) \sim_{h}^{*}(y, b)$ if and only if $\lambda\left(n, n^{\prime}\right)=0$ and $\lambda\left(n^{\prime}, n\right)=0$. As such, $\lambda$ is a not a utility representation, and yet it may provide useful information to a policy-maker who needs to trade-off costs and benefits of a policy.

Utility representations. Although, in many cases, the heuristic measure $\lambda$ may be sufficient for policy-analysis, it may also be desirable for a policy-maker (or analyst) to have a utility representation for anticipated future rankings. ${ }^{20}$

Since $\succsim_{h}^{*}$ satisfies an upper semicontinuity property, it may indeed have such a representation. In particular, let $\succsim_{h, a}^{*}$ denote the ranking with continuation problem fixed at $a$; that is, $x \succsim_{h, a}^{*} y$

\footnotetext{
${ }^{19}$ As discussed in Section 4.1, we omit time subscript for $\triangleright^{1}$ and $\triangleright^{2}$, and assume that the dominance relations satisfy the strict partial order, local non-satiation and upper semicontinuity properties.

${ }^{20}$ For instance, an upper semicontinuous utility function $u_{h}$ can be important to find an optimal solution when choice sets in period $h$ are compact but not necessarily finite.
} 
if $(x, a) \succsim_{h}^{*}(y, a)$ for all $a$. Then, $\succsim_{h, a}^{*}$ is an upper semicontinuous weak order over $X_{|h|}$. As a result, when $X_{|h|}$ is second-order countable, $\succsim_{h, a}^{*}$ can be represented by an upper semicontinuous utility function such that $x \succsim_{h, a}^{*} y$ if and only if $u_{h, a}(x) \geq u_{h, a}(y)$ (see Rader [1963]; Bosi and Mehta [2002]). ${ }^{21}$ Moreover, under additional conditions, the collection of utilities $u_{h, a}$ can be extended to an upper semicontinuous utility representation $u_{h}$ on the whole domain of $\succsim_{h}^{*}{ }^{22}$

Tie-breaking rules. Although Theorem 2 shows that a $\triangleright$-regular ranking system $\left(\succsim_{H}\right)$ can be identified uniquely, there are generally multiple plans $\varphi \in \Phi\left(\succsim_{H}\right)$ that are rationalized by the same ranking system $\left(\succsim_{H}\right) .{ }^{23}$ The reason for this multiplicity is that plans also depend on how ties are broken when indifference occurs. Understanding tie-breaking rules can be important in applied models of changing tastes. For instance, it is well-known that tie-breaking rules can lead to non-existence of a solution to a consistent planner's problem (see, e.g., Peleg and Yaari [1973]; Gul and Pesendorfer [2005]; Caplin and Leahy [2005]). ${ }^{24}$

We now consider two alternative tie-breaking rules. Let $\succsim$ be rationalized by a $\triangleright$-regular ranking system $\left(\succsim_{H}\right)$. We say that $\succsim$ is optimistic (respectively, pessimistic) if, for any plan $\varphi \in \Phi\left(\succsim_{H}\right)$, $(h, a) \succsim \varphi(h, a)$ (respectively, $\varphi(h, a) \succsim(h, a))$ for every node $(h, a) \in N$. As such, an optimistic (respectively, pessimistic) agent always anticipates that ties will be broken in her favor (respectively, against her). The following axioms provide simple conditions to identify optimism and pessimism about anticipated tie-breaking.

Axiom 4. (i) [Optimism] For all $h \in H,(x, a) \in X_{|h|} \times \mathcal{X}_{|h|+1}$, and $c \in \mathcal{X}_{|h|},(h, x, a) \succ(h, c \cup\{(x, a)\})$ implies $(h, z, a) \succ(h, c \cup\{(z, a)\})$ for some $z \in X_{|h|}$ with $z \triangleright x$.

(ii) [Pessimism] For all $h \in H,(x, a) \in X_{|h|} \times \mathcal{X}_{|h|+1}$, and $c \in \mathcal{X}_{|h|},(h, c \cup\{(x, a)\}) \succ(h, x, a)$ implies $(h, c \cup\{(z, a)\}) \succ(h, z, a)\}$ for some $z \in X_{|h|}$ with $z \triangleright x$.

Axiom 4(i) reflects that the agent anticipates ties will be broken in favor of today's preferences. In particular, whenever she anticipates that a strictly preferred alternative $(x, a)$ is not chosen from $c \cup\{(x, a)\}$, then she also anticipates that $(x, a)$ will be ranked strictly worse than some other alternative in $c$ following history $h$; otherwise, she would anticipate choosing $(x, a)$. Since her

\footnotetext{
${ }^{21}$ Such a utility representation $u_{h, a}$ may already be sufficient to find solutions for the optimization problems on a choice set with some additional structure. For instance, when the choice set is a finite union of compact subsets, where in each subset alternatives differ only in the current outcomes, and not the continuation problem.

${ }^{22}$ There are alternative conditions that suffice for an extension to a utility $u_{h}$. For instance, if $\succsim_{h}^{*}$ satisfies a stronger upper semi-continuity condition, where the set $\left\{(y, b) \in X_{|h|} \times \mathcal{X}_{|h|+1}:(x, a) \succ_{h}^{*}(y, b)\right\}$ is open for all $(x, a) \in X_{|h|} \times \mathcal{X}_{|h|+1}$, then a utility representation $u_{h}$ exists by Theorem 1 in Rader [1963]. Alternatively, if a substitution condition (i.e., whenever $a \neq b$ there exists $x, y$ such that $\left.(x, a) \sim_{h}^{*}(y, b)\right)$ and separability condition (i.e., $(x, a) \succsim_{h}^{*}(y, a)$ implies $\left.(x, b) \succsim^{*}(y, b)\right)$ are satisfied, then $u_{h, a}$ can be easily extended to a utility representation $u_{h}$ by defining $u_{h}(x, a)=u_{h, a}(x)$ for all $x$ and $a$. We also note that, when $X_{|h|}$ is metrizable, a heuristic measure $\lambda$ could be used to construct a utility function $u_{h}$ representing $\succsim_{h}$ if, in addition to conditions (i) and (ii) in the previous discussion, $\lambda$ satisfies also condition (iii) $\lambda\left(n, n^{\prime}\right)+\lambda\left(n^{\prime}, n^{\prime \prime}\right)=\lambda\left(n, n^{\prime \prime}\right)$ for any nodes $n, n^{\prime}, n^{\prime \prime}$ with a common longest history $h$.

${ }^{23}$ Note that the revealed ranking system $\left(\succsim_{H}^{*}\right)$ is identified independently of the agent's anticipated tie-breaking rule. By contrast, the canonical ranking in GP (extended to a richer setting as in Proposition 1), depends directly on the tie-breaking rule.

${ }^{24}$ Note that, in our framework with finite choice problems, consistent plans always exist. For a discussion of conditions ensuring existence in more general choice problems, see footnote 21.
} 
anticipated ranking system is regular, this means $x$ could be replaced with some $z \triangleright x$, without altering future choices. Axiom 4(ii) has an analogous interpretation, but for an agent who is pessimistic about tie-breaking.

The following result shows that these conditions characterize regular optimistic and pessimistic consistent preferences.

Proposition 2. A regular consistent preference $\succsim$ is optimistic (respectively, pessimistic) if and only if it satisfies Axiom 4(i) (respectively, Axiom 4(ii)).

\subsection{Application: elicitation of anticipated present-bias}

In this section, we present an application of our main findings on elicitation of anticipated rankings in the context of a simple parametric example with changing tastes. Eliciting anticipated behavioral parameters could be especially useful in designing economic policies (e.g., the Saving Gateway program), as well as predicting their impact and evaluating their success. ${ }^{25}$

Consumption with present-bias. Consider a three-period consumption-saving problem of a consistent planner with present-bias. Let $c_{t} \in \mathbb{R}_{+}$denote consumption in period $t=1,2{ }^{26}$ Suppose that each consumption set in period 2 is singleton, and so there is no choice of consumption in period 2. As such, any consumption plan available in period 1 is of the form $c=\left(c_{1}, c_{2}\right) \in \mathbb{R}_{+}^{2}$. Suppose that the agent is optimistic, anticipating that ties will be broken in favor of period 0 preferences (see Section 4.3).

Present-bias induces the agent's tastes to change over time. In particular, suppose the agent's preferences over consumption streams $\left(c_{1}, c_{2}\right)$ are represented by the function $v_{0}\left(c_{1}, c_{2}\right) \equiv c_{1}+c_{2}$. Moreover, in period 0 the agent anticipates making choices over consumption plans in period 1 with a utility function $v_{1}\left(c_{1}, c_{2}\right) \equiv c_{1}+\beta c_{2}$, where $\beta \in(0,1)$ is the anticipated present-bias parameter. ${ }^{27}$ How can an analyst elicit the agent's anticipated present-bias $\beta$ ?

Misidentification problem. Since the agent is a consistent planner, her period 0 preferences over consumption problems would reveal how she anticipates choosing a consumption plan from each consumption set in period 1. In particular, GP's canonical ranking rationalize the agent's preferences, and therefore an analyst could try to use Eq. (1) to identify the anticipated present-bias. However, Eq. (1) can lead to a misidentification. For instance, let $c, c^{\prime} \in \mathbb{R}_{+}^{2}$ be some consumption streams in period 1 such that $c_{1}>c_{1}^{\prime}$ while $v_{1}\left(c_{1}, c_{2}\right)=v_{1}\left(c_{1}^{\prime}, c_{2}^{\prime}\right)$; that is, $c_{1}+\beta c_{2}=c_{1}^{\prime}+\beta c_{2}^{\prime}$. Since $c_{2}=c_{2}^{\prime}-\frac{c_{1}-c_{1}^{\prime}}{\beta_{1}}<c_{2}^{\prime}$

\footnotetext{
${ }^{25}$ The present-bias model in this section could be re-interpreted in terms of the Saving Gateway example in Section 2. For instance, $c=\left(c_{1}, c_{2}\right)$ could refer to a saving policy with two dimensions (e.g., match-rate, $c_{1}$, and duration, $\left.c_{2}\right)$. While, in this re-interpreted example, today the agent would attach weight 1 to the match-rate and duration, the weights the agent would anticipate attaching to these dimensions tomorrow changes to 1 and $\beta$, respectively. As such, the agent would value the match-rate relatively more than the duration as time goes by. The standard vector-order would then provide a natural dominance relation, which allows for elicitation of the agent's anticipated future weight $\beta$ for the duration.

${ }^{26}$ To simplify the exposition, we omit consumption in period 0 .

${ }^{27}$ To focus our discussions on change of tastes due to present-bias, we consider, without loss of generality, $\beta-\delta$ preferences with linear period utility and discount factor $\delta=1$.
} 
and $\beta<1$, it follows that $c_{1}+c_{2}<c_{1}^{\prime}+c_{2}^{\prime}$, and so $v_{0}\left(c_{1}, c_{2}\right)<v_{0}\left(c_{1}^{\prime}, c_{2}^{\prime}\right)$. Moreover, since the agent is optimistic, the analyst would observe $\left(c_{1}^{\prime}, c_{2}^{\prime}\right) \sim\left(\left\{\left(c_{1}^{\prime}, c_{2}^{\prime}\right),\left(c_{1}, c_{2}\right)\right\}\right) \succ\left(c_{1}, c_{2}\right)$ in period 0 , and using Eq. (1) would incorrectly infer that $v_{1}\left(c_{1}^{\prime}, c_{2}^{\prime}\right)>v_{1}\left(c_{1}, c_{2}\right)$. As a result, the analyst would conclude that $\beta>\frac{c_{1}-c_{1}^{\prime}}{c_{2}^{\prime}-c_{2}}$, which is, however, an overestimation of the agent's actual anticipated present bias. How can the analyst overcome this problem?

Elicitation of anticipated present-bias. Notice that agent's period 0 preferences are not only consistent but are also regular. In particular, since $v_{1}$ is continuous and monotone with respect to the usual order, Axiom 3 holds. As a result, our Theorem 2 applies, and thus the agent's anticipated rankings can be elicited using Definition 1 . To illustrate, fix some $\tilde{c}_{2}>0$. Since $v_{1}$ is monotone and continuous, there is a unique consumption in period $1, \hat{c}_{1}>0$ such that $v_{1}\left(0, \tilde{c}_{2}\right)=v_{1}\left(\hat{c}_{1}, 0\right) .{ }^{28}$ Moreover, $\hat{c}_{1}$ satisfies, for any $c_{1} \in \mathbb{R}_{+}$, (i) $c_{1}<\hat{c}_{1}$ implies $\left(\left\{\left(c_{1}^{\prime}, 0\right),\left(0, \tilde{c}_{2}\right)\right\}\right) \nsim\left(c_{1}^{\prime}, 0\right)$ for some $c_{1}^{\prime}>c_{1}$, (ii) $c_{1}>\hat{c}_{1}$ implies $\left(\left\{\left(c_{1}, 0\right),\left(c_{1}^{\prime \prime}, \tilde{c}_{2}\right)\right\}\right) \nsim\left(c_{1}^{\prime \prime}, \tilde{c}_{2}\right)$ for some $c_{1}^{\prime \prime}>0$, and (iii) $c_{1}=\hat{c}_{1}$ implies both $\left(\left\{\left(c_{1}^{\prime}, 0\right),\left(0, \tilde{c}_{2}\right)\right\}\right) \sim\left(c_{1}^{\prime}, 0\right)$ for all $c_{1}^{\prime}>c_{1}$, and $\left(\left\{\left(c_{1}, 0\right),\left(c_{1}^{\prime \prime}, \tilde{c}_{2}\right)\right\}\right) \sim\left(c_{1}^{\prime \prime}, \tilde{c}_{2}\right)$ for all $c_{1}^{\prime \prime}>0$.

As such, any candidate $c_{1} \in \mathbb{R}_{+}$for the true value of $\hat{c}_{1}$ will satisfy that, when $\left(\left\{\left(c_{1}^{\prime}, 0\right),\left(0, \tilde{c}_{2}\right)\right\}\right) \nsim$ $\left(c_{1}^{\prime}, 0\right)$ for some $c_{1}^{\prime}>c_{1}$, then $\left(0, \tilde{c}_{2}\right) \succ_{1}^{*}\left(c_{1}, 0\right)$ by Definition 1 , and so $\beta \geq \frac{c_{1}}{\tilde{c}_{2}}$. The choice behavior observed in period 0 therefore provides a lower bound for $\beta$, and indicates that period 1 consumption should be increased in the choice experiment since it must be $\hat{c}_{1}>c_{1}$. On the other hand, when $\left(\left\{\left(c_{1}, 0\right),\left(c_{1}^{\prime \prime}, \tilde{c}_{2}\right)\right\}\right) \nsim\left(c_{1}^{\prime \prime}, \tilde{c}_{2}\right)$ for some $c_{1}^{\prime \prime}>0$, then $\left(c_{1}, 0\right) \succ_{1}^{*}\left(0, \tilde{c}_{2}\right)$ by Definition 1 , and so $\beta \leq \frac{c_{1}}{\tilde{c}_{2}}$. In this case, the choice behavior observed in period 0 provides an upper bound for $\beta$, and indicates that consumption in period 1 should be decreased since it must be $\hat{c}_{1}<c_{1}$. Following this procedure, one can narrow the possible range for $\beta$ with successive period 0 choice experiments. In the limit, such a procedure will lead to the candidate $c_{1}=\hat{c}_{1}$, at which both $\left(\left\{\left(c_{1}^{\prime}, 0\right),\left(0, \tilde{c}_{2}\right)\right\}\right) \sim\left(c_{1}^{\prime}, 0\right)$ for all $c_{1}^{\prime}>c_{1}$, and $\left(\left\{\left(c_{1}, 0\right),\left(c_{1}^{\prime \prime}, \tilde{c}_{2}\right)\right\}\right) \sim\left(c_{1}^{\prime \prime}, \tilde{c}_{2}\right)$ for all $c_{1}^{\prime \prime}>0$. These conditions imply that $\left(c_{1}, 0\right) \sim_{1}^{*}\left(0, \tilde{c}_{2}\right)$ by Definition 1 , and so $\beta=\frac{\hat{c}_{1}}{\tilde{c}_{2}}$, providing an accurate estimate of anticipated present-bias $\beta$.

\section{$5 \quad$ Related literature}

Consistent planning for changing tastes was introduced in Strotz [1956] and has subsequently found many applications in the literature. ${ }^{29}$ Usually, the agent's anticipated future rankings are treated as a primitive, but anticipations are, in general, not observable. Rather, predicting future behavior and measuring its welfare and policy consequences requires a method to identify anticipated rankings from choice data. Below, we discuss how we contribute to this line of research.

\footnotetext{
${ }^{28}$ Without loss of generality, we normalize consumption streams $\tilde{c}$ and $\hat{c}$ such that $\tilde{c}_{1}=\hat{c}_{2}=0$. The elicitation procedure described below is easily generalized to arbitrary consumption streams, but the normalization allows us to directly interpret $\hat{c}_{1}$ as the willingness-to-pay in period 1 for consumption $\tilde{c}_{2}$ in period 2 .

${ }^{29}$ For example, consistent planning has been used to study present-bias (e.g., O'Donoghue and Rabin [1999]; Laibson [1997]), addiction (Becker and Murphy [1988]; Becker et al. [1994]), or reference-dependence (Thaler and Benartzi [2004]; Barberis and Xiong [2009]).
} 
Consistent planning. Strotz [1956] and Pollak [1968] consider a consistent planning model with a specific structure on anticipated rankings. In particular, their primitive is a system of utility functions over outcome paths, representing changing tastes (see, e.g., Section 4.4), and the agent anticipates being a consistent planner in future periods.

Peleg and Yaari [1973] observe that the Strotz-Pollak model imposes a recursive tie-breaking rule that can lead to non-existence of a solution to the consistent planning problem. In response, they propose a generalization of the model by re-interpreting the dynamic choice problem as a game between multiple selves and considering the set of subgame perfect equilibria as potential solutions.

GP also generalize the consistent planning model by defining future rankings over continuation problems, rather than outcome paths, while also imposing that ties are broken only in favor of today's commitment ranking (i.e., optimistic tie-breaking; see Section 4.3).

All of these models are special cases of the consistent planning model we consider in this paper. In particular, apart from mild regularity conditions, we remain agnostic about the nature of anticipated future rankings or tie-breaking rules. As such, our identification approach can be applied to a wide variety of consistent planning models with changing tastes.

Identification and dominance. Gul and Pesendorfer [2001] consider preferences over decision problems to study changing tastes in the context of temptations and self-control. In particular, Gul and Pesendorfer [2001] focus on a two-period framework where decision problems consist of lottery outcomes. In this framework, they provide conditions that characterize a consistent planner. However, the future ranking that they construct is, in general, not unique; that is, there can be many ranking systems rationalizing period 0 preferences (see Abe [2012]). ${ }^{30}$

Schenone [2016] addresses the issue of separating tastes, beliefs, and states in the subjective state model of Dekel et al. [2001]. To resolve this identification problem, he adds a "monetary" dimension into the outcome space, which allows him to unambiguously rank some payoff vectors by varying this additional dimension independently of the state. Our approach to identification of changing tastes is similar in spirit in that we use a dominance relation to provide an unambiguous ranking of some alternatives independently of the history.

Siniscalchi [2011] studies consistent planning in a dynamic model of ambiguity aversion. To elicit the agent's anticipated future rankings, he assumes that, while the agent's beliefs change over time, her tastes are stable. Similar to the dominance relations in our framework, the assumption that tastes do not change over time provides a way to rank some alternatives unambiguously.

Nishimura, Ok, and Quah [2017] generalize Afriat's Theorem by using a dominance relation, which provides an objective ranking of some alternatives in their static choice framework. The key step in their result extends - by using a non-constructive argument - the dominance relation to a weak order that rationalizes choices. ${ }^{31}$

\footnotetext{
${ }^{30}$ The construction in Gul and Pesendorfer [2001] uses the canonical ranking defined in Eq. (1). Abe [2012], using a non-constructive argument, provides a different ranking that also rationalizes the same preference.

${ }^{31}$ In particular, they first construct a partial order using the dominance relation, and then extend this partial order by appealing to Szpilrajn's Lemma.
} 
Sophistication and naïveté. For policy analysis, it can be important to measure the sophistication of an agent; that is, how close the agent is to correctly anticipating her own future behavior (see, e.g., O'Donoghue and Rabin [1999]; Spiegler [2011]; Eliaz and Spiegler [2006]; Duflo et al. [2011]). To measure sophistication, one can study the evolution of preferences (or choices) over time, comparing anticipated and realized future behavior (see, e.g., Noor [2011]; Ahn et al. [2018]). ${ }^{32}$ In this regard, our identification results can be useful for measuring sophistication and naïveté of time inconsistent agents, by providing a procedure to elicit anticipated rankings from preferences in an earlier period. ${ }^{33}$

Dynamic choice and learning. In addition to changing tastes, information can be an important source for changes in future rankings. For instance, by considering preferences over dynamic decision problems, Krishna and Sadowski [2014] study a model where the agent anticipates learning about objective states, which affect her subjective tastes in the future, while Piermont et al. [2016] study a model where the agent anticipates experimenting in the future in order to learn about her tastes. In both of these models learning can induce a strict desire for flexibility, which is an attitude that does not arise in our model of consistent planning. In contrast, our model of changing tastes induces a desire for commitment, which is reflected in the no-compromise condition (Axiom 2). ${ }^{34}$

While we do not explicitly explore learning dynamics in our analysis, our results are consistent with future rankings that can accommodate learning. In particular, note that anticipated future rankings are, in general, not required to satisfy Axiom 2, and may therefore exhibit a strict desire for either flexibility or commitment.

Desire for commitment. There are various other models that also induce a desire for commitment, but which may violate Axiom 2. For instance, the random Strotz model in Dekel and Lipman [2012], the Strotz model with random tie-breaking in Caplin and Leahy [2005], the model of costly self-control in Gul and Pesendorfer [2001, 2004], or the model of costly self-regulation in Mihm and Ozbek [2018], do not necessarily satisfy the no-compromise condition. On the other hand, often in application of these models, it is natural to assume that there are dominance relations whereby future rankings are monotone. We believe that investigating whether our identification approach could be applied to such models is a potentially interesting avenue for further research. ${ }^{35}$

\footnotetext{
${ }^{32}$ For example, to measure mispredictions about future behavior, DellaVigna and Malmendier [2006] compare the choice of gym contracts - which provides information about anticipated future behavior - with actual usage of the gym in future periods.

${ }^{33}$ Choice experiments over menus are not the only method to elicit anticipated rankings and measure sophistication. For instance, in an incentivized experiment, Augenblick and Rabin [2018] directly ask subjects to bet on how many unpleasant tasks they will be willing to perform in future periods, and then compare subjects' past predictions with the actual willingness to perform these tasks.

${ }^{34}$ Learning can also be important in environments where changing tastes induce a desire for commitment, especially when an agent has the opportunity to understand that she was wrong in her prior anticipations (see, e.g., Ali [2011]).

${ }^{35}$ For instance, in a setting without lotteries, identification can remain an issue in the costly self-control model. As such, our general identification approach could prove useful as an alternative to the approach in Gul and Pesendorfer [2001], which relies on a linear structure for identification.
} 


\section{A Appendix}

In this section, we first provide two preliminary results (Lemma 1 and Lemma 2), which we invoke frequently in our formal arguments. We then provide proofs for all results stated in the paper.

\section{A.1 Preliminaries}

Let $Z$ be an arbitrary set and, as before, denote by $K(Z)$ the collection of all non-empty finite subsets of $Z$. Let $\succcurlyeq$ be binary relation defined on $K(Z)$, with the symmetric part denoted $\sim$ and the negation of the symmetric part denoted $\nsim$. We say that $\succcurlyeq$ is (i) a weak order if it is complete and transitive, (ii) satisfies no-compromise if, for all $a, b \in K(Z), a \cup b \sim a$ or $a \cup b \sim b$, and (iii) is cyclic if there are $z_{1}, z_{2}, \ldots, z_{k} \in Z$ such that, for all $i=1, \ldots, k-1,\left\{z_{i}, z_{i+1}\right\} \nsim\left\{z_{i}\right\}$ and $\left\{z_{k}, z_{1}\right\} \nsim\left\{z_{k}\right\}$.

Lemma 1. If $\succcurlyeq$ is a weak order that satisfies no-compromise, then $\succcurlyeq$ is not cyclic.

Proof. Let $\succcurlyeq$ be a weak order satisfying no-compromise and suppose, for contradiction, that $\succcurlyeq$ is cyclic: there is a finite collection $z_{1}, z_{2}, \ldots, z_{k} \in Z$ such that, for all $i=1, \ldots, k-1,\left\{z_{i}, z_{i+1}\right\} \nsim\left\{z_{i}\right\}$ and $\left\{z_{k}, z_{1}\right\} \nsim z_{k}$. Consider set $a=\left\{z_{1}, z_{2}, \ldots, z_{k}\right\}$. Since $\succcurlyeq$ satisfies no-compromise, we must have $k \geq 3$ and clearly at least two alternatives $z_{i}, z_{j} \in a$ satisfy $\left\{z_{i}\right\} \nsim\left\{z_{j}\right\}$. Thus, we can decompose $a$ into two non-empty subsets $b$ and $c$ such that for all $z \in b$, we have $a \sim\{z\}$ and for all $z \in c$ we have $a \nsim\{z\}$. Clearly, $a \sim b$ and $a \nsim c$ must hold by no-compromise. Moreover, by using sets $b$ and $c$, set $a$ can be written as $a=\left(\bigcup_{z_{j} \in b}\left\{z_{j}, z_{j+1}\right\}\right) \cup c$ where indices are modulo $k$. Now since $a \nsim c$, by no-compromise, we must have $a \sim\left\{z_{j}, z_{j+1}\right\}$ for some $z_{j} \in b$. But this is a contradiction because $a \sim\left\{z_{j}\right\} \nsim\left\{z_{j}, z_{j+1}\right\}$.

In the following lemma, let $\succsim$ be a preference relation on $X_{0} \times \mathcal{X}_{1}$ and let $\left(\succsim_{H}^{*}\right)$ be its revealed ranking system (Definition 1 ).

Lemma 2. If $\succsim$ satisfies Axioms 1 , 2 and 3, then $\left(\succsim_{H}^{*}\right)$ is a $\triangleright$-regular ranking system.

Proof. Let $\succsim$ be a preference relation satisfying Axioms 1, 2 and 3. Fix some history $h \in H$, and consider the corresponding history-dependent revealed ranking $\succsim_{h}^{*}$. The proof follows by showing that $\succsim_{h}^{*}$ is complete, transitive, monotone and upper semicontinuous.

Step $1\left[\succsim_{h}^{*}\right.$ is complete]: Suppose, for contradiction, $\succsim_{h}^{*}$ is not complete. In that case, there must exist some $x, y \in X_{|h|}$ and $a, b \in \mathcal{X}_{|h|+1}$ such that $\left(h,\left\{\left(x^{\prime}, a\right),(y, b)\right\}\right) \nsim\left(h, x^{\prime}, a\right)$ for some $x^{\prime} \in X_{|h|}$ with $x^{\prime} \triangleright x$ and $\left(h,\left\{(x, a),\left(y^{\prime}, b\right)\right\}\right) \nsim\left(h, y^{\prime}, b\right)$ for some $y^{\prime} \in X_{|h|}$ with $y^{\prime} \triangleright y$. By Axiom $3(\mathrm{i}),\left(h,\left\{(x, a),\left(x^{\prime}, a\right)\right\}\right) \nsim(h, x, a)$ and $\left(h,\left\{(y, b),\left(y^{\prime}, b\right)\right\}\right) \nsim(h, y, b)$ implying $\succsim$ is cyclic, which contradicts with Axioms 1 and 2 by Lemma 1. Thus, $\succsim_{h}^{*}$ must be complete.

Step $2 \succsim_{h}^{*}$ is transitive]: Let $(x, a),(y, b),(z, c) \in X_{|h|} \times \mathcal{X}_{|h|+1}$ such that $(x, a) \succsim_{h}^{*}(y, b)$, $(y, b) \succsim_{h}^{*}(z, c)$. We want to show that $(x, a) \succsim_{h}^{*}(z, c)$. To do this, we first show that for any $x^{\prime} \in X_{|h|}$ with $x^{\prime} \triangleright x$, there exists some $y^{\prime} \in X_{|h|}$ with $y^{\prime} \triangleright y$ such that $\left(h,\left\{\left(x^{\prime}, a\right),\left(y^{\prime}, b\right)\right\}\right) \nsim\left(h, y^{\prime}, b\right)$. Otherwise, there exists some $x^{\prime} \triangleright x$ such that $\left(h,\left\{\left(x^{\prime}, a\right),\left(y^{\prime}, b\right)\right\}\right) \sim\left(h, y^{\prime}, b\right)$ for all $y^{\prime} \triangleright y$. But in this case, for all $x^{\prime \prime} \in X_{|h|}$ with $x^{\prime} \triangleright x^{\prime \prime},\left(h,\left\{\left(x^{\prime \prime}, a\right),(y, b)\right\}\right) \sim(h, y, b)$ by Axiom 3(ii), which 
contradicts with the fact that $(x, a) \succsim_{h}^{*}(y, b)$. Now given that $\left(h,\left\{\left(x^{\prime}, a\right),\left(y^{\prime}, b\right)\right\}\right) \nsim\left(h, y^{\prime}, b\right)$, we can assume, without loss of generality, that $y^{\prime}$ satisfies $\left(h, y^{\prime}, b\right) \nsim(h, z, c)$. Otherwise, one can always find some other $y^{\prime \prime} \in X_{|h|}$ such that $y^{\prime} \triangleright y^{\prime \prime} \triangleright y$ and $\left(h,\left\{\left(x^{\prime}, a\right),\left(y^{\prime \prime}, b\right)\right\}\right) \nsim\left(h, y^{\prime \prime}, b\right)$, and use $y^{\prime \prime}$ instead of $y^{\prime}$. By definition of $\succsim_{h}^{*}$, we must have $\left(h,\left\{\left(y^{\prime}, b\right),(z, c)\right\}\right) \nsim(h, z, c)$ implying that $\left(h,\left\{\left(x^{\prime}, a\right),(z, c)\right\}\right) \sim\left(h, x^{\prime}, a\right)$. Otherwise, $\succsim$ would be cyclic contradicting with Axioms 1 and 2 by Lemma 1. Since $x^{\prime} \in X_{|h|}$ is arbitrary, we conclude that $(x, a) \succsim_{h}^{*}(z, c)$, showing $\succsim_{h}^{*}$ is transitive.

Step $3\left[\succsim_{h}^{*}\right.$ is monotone]: Let $a \in \mathcal{X}_{|h|+1}$, and let $x, y, z \in X_{|h|}$ with $x \triangleright z \triangleright y$. Then by Axiom $3(\mathrm{i})$, we have $(h,\{(x, a),(z, a)\}) \nsim(h, z, a)$ showing that $(x, a) \succ_{h}^{*}(y, a)$, and so $\succsim_{h}^{*}$ is monotone.

Step $4 \succsim_{h}^{*}$ is upper semi-continuous]: We want to show $A=\left\{y \in X_{|h|}:(y, b) \succsim_{h}^{*}(x, a)\right\}$ is a closed set for any $(x, a) \in X_{|h|} \times \mathcal{X}_{|h|+1}$ and $b \in \mathcal{X}_{|h|+1}$. To do this, let $\left\{y_{k}\right\}$ be a sequence in $A$ such that $y_{k} \rightarrow y$. Using upper semicontinuity of $\triangleright$, for any $y^{\prime} \in X_{|h|}$ with $y^{\prime} \triangleright y$ there exists some $y_{k^{\prime}} \in\left\{y_{k}\right\}$ such that $y^{\prime} \triangleright y_{k^{\prime}}$. Otherwise $\left\{y_{k}\right\}$ can not approach $y$. Since $\left(y_{k^{\prime}}, b\right) \succsim_{h}^{*}(x, a)$, and $y^{\prime} \triangleright y_{k^{\prime}}$, we have $\left(y^{\prime}, b\right) \succ_{h}^{*}(x, a)$ using monotonicity and transitivity of $\succsim_{h}^{*}$. By definition of $\succsim_{h}^{*}$, there must exist some $x^{\prime} \in X_{|h|}$ with $x^{\prime} \triangleright x$ such that $\left(h,\left\{\left(y^{\prime}, b\right),\left(x^{\prime}, a\right)\right\}\right) \nsim\left(h, x^{\prime}, a\right)$, and so $\left(h,\left\{\left(y^{\prime}, b\right),(x, a)\right\}\right) \sim\left(h, y^{\prime}, b\right)$. Otherwise, since we have $\left(h,\left\{\left(x^{\prime}, a\right),\left(x^{\prime}, a\right)\right\}\right) \nsim(h, x, a)$ by Axiom $3(\mathrm{i}), \succsim$ would be cyclic contradicting Axioms 1 and 2 by Lemma 1 . Since $y^{\prime} \in X_{|h|}$ is arbitrary, we conclude that $(y, b) \succsim_{h}^{*}(x, a)$ showing $A$ is a closed set, and so $\succsim_{h}^{*}$ is upper semi-continuous.

\section{A.2 Proof of Proposition 1}

In the following, let $R: \times_{t=1}^{T} K\left(X_{t}\right) \rightarrow K\left(X_{0} \times \mathcal{X}_{1}\right)$ be a mapping where, for finite subsets $Y_{1} \subseteq X_{1}, \ldots, Y_{T} \subseteq X_{T}$, the finite subset of initial nodes $R(Y) \subseteq X_{0} \times \mathcal{X}_{1}$ is constructed recursively as described in Section 3.1. Given $Y=\left(Y_{1}, \ldots, Y_{T}\right) \in \times_{t=1}^{T} K\left(X_{t}\right)$ and a plan $\varphi \in \Phi$, we denote by $\varphi_{Y}$ the restriction of the plan $\varphi$ to the finite set of initial nodes $R(Y)$, and by $\left(\succsim_{H \mid Y}\right)$ a ranking system defined on the restricted domain of initial nodes $R(Y)$.

Proof. The necessity of the axioms is straightforward, and so we omit this direction of the proof. To show that the axioms are sufficient, let $\succsim$ be a binary relation on $X_{0} \times \mathcal{X}_{1}$ satisfying Axioms 1 and 2. By iteratively applying Axiom 2, it is straightforward to show that $\succsim$ is consistent with a plan $\varphi \in \Phi$, and it remains to show that this plan can be rationalized by a ranking system $\left(\succsim_{H}\right)$.

For each $Y \in \times_{t=1}^{T} K\left(X_{t}\right)$, Theorem 2 in GP identifies a canonical ranking system $\left(\succsim_{H \mid Y}^{G P}\right)$, which rationalizes the restriction of $\varphi$ on $R(Y)$; that is, $\varphi_{Y} \in \Phi\left(\succsim_{H \mid Y}^{G P}\right)$. Moreover, for any $Y, Z \in \times_{t=1}^{T} K\left(X_{t}\right)$ with $Y_{t} \subseteq Z_{t}$ for all $t=1, \ldots, T$, the plan $\varphi_{Z}$ agrees with the plan $\varphi_{Y}$ on $R(Y)$, since $R(Y)$ is a subset of $R(Z)$, and therefore the ranking system $\left(\succsim_{H \mid Z}^{G P}\right)$ agrees with the ranking system $\left(\succsim_{H \mid Y}^{G P}\right)$ on $R(Y)$. In that case, we say that $\left(\succsim_{H \mid Z}^{G P}\right)$ extends $\left(\succsim_{H \mid Y}^{G P}\right)$, denoted $\left(\succsim_{H \mid Z}^{G P}\right) \ni\left(\succsim_{H \mid Y}^{G P}\right)$. By definition, the binary relation $\ni$ is a partial order, and the following class of ranking systems $\mathcal{R}=\left\{\left(\succsim_{H \mid Y}^{G P}\right) \mid Y \in \times_{t=1}^{T} K\left(X_{t}\right)\right\}$ is therefore partially ordered by $\ni$. Thus, by the Hausdorff Maximal Principle, there exists a maximal chain (i.e., a totally ordered subset) in $\mathcal{R}$ (see, e.g., Royden [1988, p. 25] or Suppes [1972, p. 248]). Pick one such maximal chain, and call it $\mathcal{P}$. Since $\mathcal{P}$ is totally ordered, it consists of ranking systems $\left(\succsim_{H \mid Y}^{G P}\right)$ which are monotonically extending with respect to $\ni$. 
As a result, these ranking systems converge to a ranking system $\left(\succsim_{H}\right)$ such that $\left(\succsim_{H}\right) \ni\left(\succsim_{H \mid Y}^{G P}\right)$ for any $\left(\succsim_{H \mid Y}^{G P}\right) \in \mathcal{P}$. Hence, $\left(\succsim_{H}\right)$ rationalizes the plan $\varphi \cdot{ }^{36}$

\section{A.3 Proof of Theorem 1}

Proof. We first show that when $\succsim$ satisfies Axioms 1, 2 and 3, then it is a regular consistent preference. It follows immediately from the axioms that $\succsim$ is $\triangleright$-sensitive. Moreover, by Lemma 2, its revealed ranking system $\left(\succsim_{H}^{*}\right)$ is $\triangleright$-regular. Therefore, it remains to show that $\succsim$ is compatible with a plan $\varphi \in \Phi\left(\succsim_{H}^{*}\right)$. To show this, let $(x, a),(y, b) \in X_{|h|} \times \mathcal{X}_{|h|+1}$ with $(x, a) \succ_{h}^{*}(y, b)$. By Definition 1 , there exists $y^{\prime} \in X_{|h|}$ with $y^{\prime} \triangleright y$ such that $\left(h,\left\{\left(x^{\prime}, a\right),\left(y^{\prime}, b\right)\right\}\right) \sim\left(h, x^{\prime}, a\right)$ for all $x^{\prime} \in X_{|h|}$ with $x^{\prime} \triangleright x$. Thus, $\left(h,\left\{(x, a),\left(y^{\prime \prime}, b\right)\right\}\right) \sim(h, x, a)$ for any $y^{\prime \prime} \in X_{|h|}$ such that $y^{\prime} \triangleright y^{\prime \prime}$ by Axiom 3(ii), and so $(h,\{(x, a),(y, b)\}) \sim(h, x, a)$, showing $\succsim$ is rationalized by $\left(\succsim_{H}^{*}\right)$.

For the converse, let $\succsim$ be a regular consistent preference. By definition, $\succsim$ is $\triangleright$-sensitive and it is compatible with a plan $\varphi \in \Phi\left(\succsim_{H}\right)$ rationalized by a $\triangleright$-regular ranking system $\left(\succsim_{H}\right)$. Since $\succsim$ is a consistent preference, it satisfies Axioms 1 and 2 by Proposition 1. To show that $\succsim$ satisfies Axiom 3(i), let $h \in H, a \in \mathcal{X}_{|h|+1}$, and $x, y \in X_{|h|}$ such that $x \triangleright y$. Since $\succsim_{h}$ is monotone, we have $(x, a) \succ_{h}(y, a)$, and so $(h,\{(x, a),(y, a)\}) \sim(h, x, a)$ by the fact that $\succsim$ is rationalized by $\left(\succsim_{H}\right)$. Since $\succsim$ is $\triangleright$-sensitive, $(h, x, a) \nsim(h, y, a)$. Since $\succsim$ is transitive, it follows that $(h,\{(x, a),(y, a)\}) \nsim(h, y, a)$, showing $\succsim$ satisfies Axiom 3(i).

To show that $\succsim$ satisfies Axiom 3(ii), let $h \in H,(x, a),(y, b) \in X_{|h|} \times \mathcal{X}_{|h|+1}$ such that

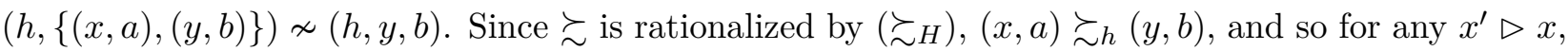
$\left(x^{\prime}, a\right) \succ_{h}(y, b)$ by monotonicity of $\succsim_{h}$. As a result, there exists $y^{\prime} \triangleright y$ such that $\left(x^{\prime}, a\right) \succ_{h}\left(y^{\prime}, b\right)$ by upper semicontinuity of $\succsim_{h}$. Since $\succsim$ is rationalized by $\left(\succsim_{H}\right),\left(h,\left\{\left(x^{\prime}, a\right),\left(y^{\prime}, b\right)\right\}\right) \sim\left(h, x^{\prime}, a\right)$. Moreover, since $\succsim$ is $\triangleright$-sensitive, without loss of generality we can choose $y^{\prime}$ such that $\left(h, x^{\prime}, a\right) \nsim\left(h, y^{\prime}, b\right)$, and so $\left(h,\left\{\left(x^{\prime}, a\right),\left(y^{\prime}, b\right)\right\}\right) \nsim\left(h, y^{\prime}, b\right)$ by transitivity of $\succsim$.

\section{A.4 Proof of Theorem 2}

Proof. Suppose $\succsim$ is $\triangleright$-sensitive and can be rationalized by a $\triangleright$-regular ranking system $\left(\succsim_{H}\right)$. We want to show that $\left(\succsim_{H}\right)=\left(\succsim_{H}^{*}\right)$. By Theorem 1, $\succsim$ satisfies Axioms 1, 2 and 3. As such, by Lemma 2 , its revealed ranking system $\left(\succsim_{H}^{*}\right)$ is $\triangleright$-regular. Moreover, as we show in the proof of Theorem 1 , $\succsim$ can be rationalized by $\left(\succsim_{H}^{*}\right)$.

Now suppose for contradiction that there is some $h \in H$, and some $(x, a),(y, b) \in X_{|h|} \times \mathcal{X}_{\mid h+1}$ such that $(x, a) \succsim_{h}(y, b)$ while $(y, b) \succ_{h}^{*}(x, a)$. Since $\succsim_{h}$ and $\succsim_{h}^{*}$ satisfy monotonicity and upper semicontinuity, and $\succsim$ is $\triangleright$-sensitive, one can assume without loss of generality that $(x, a) \succ_{h}(y, b)$ and $(h, x, a) \nsim(h, y, b)$. Since $\succsim$ is rationalized by both $\left(\succsim_{H}^{*}\right)$ and $\left(\succsim_{H}\right)$, we must have $(h, x, a) \sim$ $(h,\{(x, a),(y, b)\}) \sim(h, y, b)$, a contradiction.

\footnotetext{
${ }^{36}$ Note that $\left(\succsim_{H}\right)$ does not depend on the choice of maximal chain $\mathcal{P}$ because, eventually, any two such maximal chains must have the same sequence of extending ranking systems; otherwise, none of them are a maximal chain.
} 


\section{A.5 Proof of Theorem 3}

Proof. For $j=1,2$, let $\left(\succsim_{H}^{j}\right)$ be a $\triangleright^{j}$-regular ranking system, and let $\succsim$ be a $\triangleright^{j}$-sensitive preference relation that can be rationalized by $\left(\succsim_{H}^{j}\right)$. For contradiction, suppose there is some $h \in H, x, y \in X_{|h|}$ and $a, b \in \mathcal{X}_{|h|+1}$ such that $(x, a) \succ_{h}^{1}(y, b)$ and $(y, b) \succsim_{h}^{2}(x, a)$. Since the ranking system $\left(\succsim_{H}^{1}\right)$ is $\triangleright^{1}$-regular (in particular, upper semicontinuous), there exists an open neighborhood $O_{y}$ around $y$ such that for all $y^{\prime} \in O_{y}$, we have $(x, a) \succ_{h}^{1}\left(y^{\prime}, b\right)$. Since $\triangleright^{2}$ is locally non-satiated, there exists $z \in O_{y}$ such that $z \triangleright^{2} y$. Moreover, since $\succsim$ is $\triangleright^{2}$-sensitive, without loss of generality, we can choose $z$ such that $(h, x, a) \nsim(h, z, b)$. Since $\left(\succsim_{H}^{2}\right)$ is $\triangleright^{2}$-regular (in particular, monotone), we have $(z, b) \succ_{h}^{2}(x, a)$. As such, we have $(h,\{(x, a),(z, b)\}) \nsim(h, z, b)$ since $\left(\succsim_{H}^{1}\right)$ rationalizes $\succsim$ and $(x, a) \succ_{h}^{1}(z, b)$. On the other hand, since $\left(\succsim_{H}^{2}\right)$ rationalizes $\succsim$ and $(z, b) \succ_{h}^{2}(x, a)$ we have $(h,\{(x, a),(z, b)\}) \sim(h, z, b)$, which is a contradiction.

\section{A.6 Proof of Proposition 2}

Proof. We provide the proof for an optimistic regular consistent preference; the proof for the pessimistic case follows a similar argument. Suppose $\succsim$ is a regular consistent preference. By Theorem 1 , $\succsim$ is compatible with a plan $\varphi \in \Phi\left(\succsim_{H}^{*}\right)$, where $\left(\succsim_{H}^{*}\right)$ is the revealed ranking system. Moreover, by Theorem 2, $\left(\succsim_{H}^{*}\right)$ is the only $\triangleright$-regular ranking system that can rationalize $\varphi$.

[If $\succsim$ is optimistic, then $\succsim$ satisfies Axiom 4(i)]: Let $h \in H,(x, a) \in X_{|h|} \times \mathcal{X}_{|h|+1}, c \in \mathcal{X}_{|h|}$, and $\varphi(h, c \cup\{(x, a)\})=(y, b)$. Assume $(h, x, a) \succ(h, y, b)$. Since $\succsim$ is optimistic, it must be that $(y, b) \succ_{h}^{*}(x, a)$, otherwise we can find a plan $\varphi^{\prime} \in \Phi\left(\succsim_{H}^{*}\right)$ such that $\varphi^{\prime}(h, c \cup\{(x, a)\})=(x, a)$ and then $\varphi^{\prime}(h, c \cup\{(x, a)\}) \succ \varphi(h, c \cup\{(x, a)\})$. Since $\succsim_{h}^{*}$ is upper semicontinuous, and partial order $\triangleright$ satisfies local non-satiation, we can find some $x^{\prime} \in X_{|h|}$ such that $(y, b) \succ_{h}^{*}\left(x^{\prime}, a\right)$. Since $\varphi \in \Phi\left(\succsim_{H}^{*}\right)$, we have $\varphi\left(h, c \cup\left\{\left(x^{\prime}, a\right)\right\}\right)=(h, y, b)$. Thus, $\left(h, x^{\prime}, a\right) \succ\left(h, c \cup\left\{\left(x^{\prime}, a\right)\right\}\right)$ by Axioms 1 and 3 .

[If $\succsim$ satisfies Axiom $4(i)$, then $\succsim$ is optimistic]: We show the contrapositive. Suppose that $\succsim$ is not optimistic. In that case, there exists some plan $\varphi^{\prime} \in \Phi\left(\succsim_{H}^{*}\right)$ such that, for some node $(h, c) \in N$ such that $\varphi^{\prime}(h, c) \succ \varphi(h, c)$. Let $\varphi^{\prime}(h, c)=(h, x, a)$ and $\varphi(h, c)=(h, y, b)$. Since both plans, $\varphi^{\prime}$ and $\varphi$ are rationalized by the ranking system $\left(\succsim_{H}^{*}\right)$, it must be $(x, a) \sim_{h}^{*}(y, b)$. Let $x^{\prime} \in X_{|h|}$ such that

$x^{\prime} \triangleright x$. Since $\succsim_{h}^{*}$ is monotone, $\left(x^{\prime}, a\right) \succ_{h}^{*}(y, b)$. This implies $\varphi\left(\left(h,\left(c \cup\left\{\left(x^{\prime}, a\right)\right\}\right)\right)=\left(h, x^{\prime}, a\right)\right.$ and so $\left(h, x^{\prime}, a\right) \sim\left(h,\left(c \cup\left\{\left(x^{\prime}, a\right)\right\}\right)\right.$. Since $x^{\prime}$ was arbitrary, it follows that Axiom 4 is not satisfied.

Acknowledgement We thank the editor, an associate editor and two anonymous referees for insightful comments, which helped us to improve the paper. We also thank Faruk Gul, John Leahy, Efe Ok, Joel Sobel, Yiannis Vailakis, conference participants at FUR 2018, and seminar participants at the University of Glasgow for helpful comments and discussions.

\section{References}

ABE, K. (2012): "A Geometric Approach to Temptation," Journal of Mathematical Economics, 48, $92-97$. 
Ahn, D., R. Iijima, Y. Le Yaouang, And T. Sarver (2018): "Behavioral Characterizations of Naivete for Time-Inconsistent Preferences," Review of Economic Studies, forthcoming.

Ali, S. N. (2011): "Learning Self-Control," The Quarterly Journal of Economics, 126, 857-893.

Augenblick, N. And M. Rabin (2018): "An Experiment on Time Preference and Misprediction in Unpleasant Tasks," The Review of Economic Studies.

Barberis, N. And W. Xiong (2009): "What Drives the Disposition Effect? An Analysis of a Long-standing Preference-based Explanation," The Journal of Finance, 64, 751-784.

Becker, G. S., M. Grossman, And K. M. Murphy (1994): "An Empirical Analysis of Cigarette Addiction," American Economic Review, 84, 396-418.

Becker, G. S. And K. M. Murphy (1988): "A Theory of Rational Addiction," Journal of Political Economy, 96, 675-700.

Bosi, G. And G. B. Mehta (2002): "Existence of a Semicontinuous or Continuous Utility Function: A Unified Approach and an Elementary Proof," Journal of Mathematical Economics, 38, 311-328.

Caplin, A. And J. Leahy (2005): "The Recursive Approach to Time Inconsistency," Journal of Economic Theory, 131, 134-156.

Dekel, E. And B. L. Lipman (2012): "Costly Self-Control and Random Self-Indulgence," Econometrica, 80, 1271-1302.

Dekel, E., B. L. Lipman, and A. Rustichini (2001): "Representing Preferences with a Unique Subjective State Space," Econometrica, 69, 891-934.

Della Vigna, S. (2009): "Psychology and Economics: Evidence from the Field," Journal of Economic Literature, 47, 315-372.

Della Vigna, S. and U. Malmendier (2006): "Paying Not to Go to the Gym," American Economic Review, 96, 694-719.

Duflo, E., M. Kremer, and J. Robinson (2011): "Nudging Farmers to Use Fertilizer: Evidencefrom Kenya," American Economic Review, 101, 2350-2390.

Eliaz, K. And R. Spiegler (2006): "Contracting with Diversely Naive Agents," The Review of Economic Studies, 72, 689-714.

Emmerson, C., P. Harvey, N. Pettigrew, R. Madden, G. Tetlow, and M. Wakefield (2007): "Final Evaluation of the Saving Gateway 2 Pilot: Main Report," HM Treasury, Department for Education and Skills.

Gul, F. And W. Pesendorfer (2001): "Temptation and Self-Control," Econometrica, 69, 14031435. 
(2004): "Self-Control and the Theory of Consumption," Econometrica, 72, 119-158.

(2005): "The Revealed Preference Theory of Changing Tastes," Review of Economic Studies, $72,429-448$.

Kempson, E., S. McKay, And S. Collard (2005): "Incentives to Save: Encouraging Saving Among Low-Income Households," Personal Finance Research Center, University of Bristol.

Krishna, R. V. And P. Sadowski (2014): "Dynamic Preference for Flexibility," Econometrica, $82,655-703$.

Laibson, D. (1997): "Golden Eggs and Hyperbolic Discounting," The Quarterly Journal of Economics, 112, 443-478.

Minm, M. And K. OzBeK (2018): "Mood-driven Choices and Self-Regulation," Journal of Economic Theory, 176, 727-760.

Nishimura, H., E. A. OK, And J. K.-H. Quah (2017): "A Comprehensive Approach to Revealed Preference Theory," American Economic Review, 107, 1239-1263.

Noor, J. (2011): "Temptation and Revealed Preference," Econometrica, 79, 601-644.

O’Donoghue, T. and M. Rabin (1999): "Doing It Now or Later," American Economic Review, $89,103-124$.

- (2015): "Present Bias: Lessons Learned and To Be Learned," American Economic Review: Papers and Proceedings, 105, 273-279.

Peleg, B. And M. E. YaAri (1973): "On the Existence of a Consistent Course of Action when Tastes are Changing," The Review of Economic Studies, 40, 391-401.

Phelps, E. S. And R. A. Pollak (1968): "On Second-Best National Saving and Game-Equilibrium Growth," Review of Economic Studies, 35, 185-199.

Piermont, E., N. Takeoka, And R. Teper (2016): "Learning the Krepsian State: Exploration Through Consumption," Games and Economic Behavior, 100, 69-94.

Pollak, R. A. (1968): "Consistent Planning," The Review of Economic Studies, 35, 201-208.

RAder, T. (1963): "The Existence of a Utility Function to Represent Preferences," The Review of Economic Studies, 30, 229-232.

Royden, H. L. (1988): Real Analysis, Macmillan Publishers Ltd.

Schenone, P. (2016): "Identifying Subjective Beliefs in Subjective State Space Models," Games and Economic Behavior, 95, 59-72.

Siniscalchi, M. (2011): "Dynamic Choice under Ambiguity," Theoretical Economics, 6, 379-421. 
SPIEGler, R. (2011): Bounded Rationality and Industrial Organization, Oxford University Press.

Strotz, R. H. (1956): "Myopia and Inconsistency in Dynamic Utility Maximization," Review of Economic Studies, 23, 165-180.

Suppes, P. (1972): Axiomatic Set Theory, Dover Publications, Inc.

Thaler, R. H. And S. Benartzi (2004): "Save More Tomorrow: Using Behavioral Economics to Increase Employee," Journal of Political Economy, 112, 164-187.

Toussaert, S. (2018): "Eliciting Temptation and Self-control Through Menu Choices: A Lab Experiment," Econometrica, 86, 859-889. 